\title{
An overview of acute hypertensive response following intracerebral hemorrhage: implifications for clinical practice
}

\author{
Yasemin Akinci, Adnan I. Qureshi \\ Zeenat Qureshi Stroke Institute and Department of Neurology, University of Missouri, Columbia, MO 65212, USA. \\ Correspondence to: Dr. Adnan I. Qureshi, Zeenat Qureshi Stroke Institute and Department of Neurology, University of Missouri, \\ Columbia, MO 65212, USA. E-mail: qureshai@gmail.com
}

How to cite this article: Akinci Y, Qureshi Al. An overview of acute hypertensive response following intracerebral hemorrhage: implifications for clinical practice. Vessel Plus 2021;5:56. https://dx.doi.org/10.20517/2574-1209.2021.20

Received: 2 Feb 2021 First Decision: 17 Mar 2021 Revised: 19 Mar 2021 Accepted: 20 Apr 2021 Published: 8 Dec 2021

Academic Editor: Elisa F. M. Ciceri Copy Editor: Yue-Yue Zhang Production Editor: Yue-Yue Zhang

\begin{abstract}
The acute hypertensive response, elevated blood pressure in the acute phase of intracerebral hemorrhage, is very common and is associated with hematoma expansion, cerebral edema formation, intracranial pressure elevation, and poor long-term outcomes. This has led to the acceptance of antihypertensive treatment of acute hypertensive response as a mainstay of intacerebral hemorrhage management to reduce hematoma expansion and the associated disability and mortality. Intensive lowering of systolic blood pressure in the acute phase of intracerebral hemorrhage with varying intensity of systolic blood pressure reduction has been evaluated in several clinical trials. Some of these trials showed little benefit with intensive systolic blood pressure reduction compared with moderate or standard blood pressure reduction, while some showed no benefit and despite the large amount of literature, optimal approaches are not yet clear. In this review article, we summarized the current knowledge of the mechanisms underlying acute hypertensive response and the recent guidelines on the treatment of the acute hypertensive response provided by professional organizations and results of clinical trials and discussed incorporation of these concepts into clinical practice.
\end{abstract}

Keywords: Acute hypertensive response, intracerebral hemorrhage, hematoma expansion, blood pressure reduction, stroke 


\section{INTRODUCTION}

Intracerebral hemorrhage ( $\mathrm{ICH}$ ) is leakage of blood into the brain parenchyma with or without extension of blood into the ventricles and/or the subarachnoid space, either spontaneously or as a result of trauma. Chronic arterial hypertension, cerebral amyloid angiopathy, bleeding diathesis (congenital, acquired, induced by anticoagulants or antithrombotics), tumors, cerebral venous thrombosis, vasculitis, hemorrhagic transformation of a recent ischemic stroke, vascular malformations, stimulant drugs (cocaine, amphetamine, and ecstasy) are etiological factors of spontaneous (also known as non-traumatic) $\mathrm{ICH}^{[1-4]}$. To date, there have been no generally accepted criteria for the etiologic classification of spontaneous ICH, but since chronic arterial hypertension and cerebral amyloid angiopathy are the most common etiological factors, ICH associated with these two factors has been classified as primary ICH, while ICH attributed to other factors has been considered secondary ${ }^{[1-3,5]}$. The focus of this review is primary ICH, which is believed to result from lipohyalinosis and degenerative changes of the small penetrating arteries associated with hypertension or amyloid angiopathy with the exception of secondary ICH associated with other causes ${ }^{[2]}$. However, albeit at a low rate, patients receiving antiplatelets or anticoagulants were included in the clinical studies that we will examine in the following sections.

Primary ICH is commonly seen in the basal ganglia, thalamus, cerebral lobes, brain stem (predominantly pons), and cerebellum ${ }^{[2]}$. ICH is a multiple-stage dynamic condition including initial extravasation of blood into the parenchyma, followed by bleeding around the clot causing hematoma expansion, and edema or tissue swelling around the hemorrhage caused by pro-osmotic substances released from the acute hematoma ${ }^{[1,2]}$. The subsequent hematoma expansion after initial ictus, which occurs with a highest rate within the first $3 \mathrm{~h}$ from symptom onset and stabilizes within the first $24 \mathrm{~h}$, has been well documented by repeated brain imaging techniques in many studies and is associated with neurological deterioration, poor functional outcomes and increased mortality ${ }^{[6]}$. Previous data indicate that every $1 \mathrm{~mL}$ growth in hematoma can increase the risk of death or disability by $7 \%{ }^{[7]}$. The mechanism of hematoma expansion that therapeutic interventions are focused on, is not yet clearly understood but is likely related to inflammatory cascade upregulation resulting in imbalance in hemostatic mechanisms and increased expression of matrix metalloproteinases causing disruption of physical support by the endothelial basement membrane, stretching and microscopic rupture of surrounding vessels due to the mass effect of the clot, and intracranial pressure (ICP) causing disruption of venous outflow and subsequent vascular engorgement ${ }^{[1]}$. In addition to these alleged mechanisms, elevated systolic blood pressure (SBP), termed as acute hypertensive response, has been shown to increase both hematoma expansion and perihematomal edema in acute ICH patients at the time of hematoma and is a strong, independent predictor of mortality and disability ${ }^{[8-12]}$. This has led to the blood pressure (BP) control, which is considered as an important treatment strategy for the prevention of $\mathrm{ICH}$, also being topics of extensive studies evaluating the reduction of perihematomal edema, prevention of hematoma expansion and following mortality and disability in acute ICH patients ${ }^{[13,14]}$.

\section{ACUTE HYPERTENSIVE RESPONSE}

Acute hypertensive response is defined as elevation of SBP above normal ( $\geq 140 \mathrm{mmHg}$ ) and premorbid values that occurs in the first $24 \mathrm{~h}$ after symptom onset in patients with $\mathrm{ICH}^{[6]}$. This highly prevalent systemic response (which occurs in almost 75\% of ICH patients, regardless of prior history of hypertension), is self-limiting with spontaneous reduction within few days ${ }^{[15]}$. Chronic hypertension is the most important risk factor for spontaneous $\mathrm{ICH}^{[16]}$. Due to higher prevalence on the basis of premorbid hypertension, it can be assumed that in at least a proportion of patients, the acute hypertensive response is a reflection of poorly treated or undetected chronic hypertension, but spontaneous decrease of initial BP over the next few days in most patients suggests that other mechanisms associated with stroke itself are responsible. Proposed underlying causes of acute hypertensive response include: 
(1) Transient or permanent damage to the widely distributed areas responsible for BP regulation including prefrontal cortex, insula, amygdala, hypothalamus, cingulate cortex, and brainstem (particularly ventrolateral medulla and nucleus tractus solitarius ${ }^{[6]}$.

(2) Autonomic dysregulation caused by increased sympathetic activity due to subsequent release of renin and vasoconstriction of arterioles results from direct damage to inhibitory or modulatory brain regions, or indirect effects of decreased parasympathetic activity leading to reduced baroreceptor sensitivity and ultimately significant BP variability ${ }^{[6]}$.

(3) Raised ICP (especially with brainstem compression) due to acutely increased intracranial volume and reduced intracranial compliance (Cushing's reflex) ${ }^{[17]}$.

(4) Raised levels of circulating catecholamines and inflammatory cytokines due to stress related to hospitalization, white coat hypertension, pain, urinary retention, and concominant infection ${ }^{[17]}$.

This phenomenon is not specific to ICH, but is associated with all stroke subtypes most likely with differences in underlying pathophysiology, but among stroke subtypes the prevalence of severe hypertension is higher in acute ICH patients (in one study, the proportion of patients with initial SBP $\geq 140$ $\mathrm{mmHg}$ was $67 \%$ in ischemic stroke patients $v s .75 \%$ in ICH patients ${ }^{[15]}$. This difference is not only significant in prevalence but also in severity. The Oxford Vascular Study showed that despite similar rates of chronic hypertension history, the difference between the mean first SBP after stroke onset and the most recent pre-stroke reading was markedly greater in ICH patients than in ischemic stroke patients (mean 43.5 $\mathrm{mmHg} v$ s. mean $17.9 \mathrm{mmHg}$ ) and acute-phase SBP was much closer to the usual long-term premorbid values in major ischemic stroke patients ${ }^{[18]}$.

High diastolic blood pressure (DBP) ( $\geq 90 \mathrm{mmHg}$ ) has also been documented in patients with acute ICH (found to occur in $23.6 \%$ of patients admitted with $\mathrm{ICH}$ ). However, as the available data do not indicate a clear association between DBP and hematoma expansion, SBP is preferably used to describe the acute hypertensive response $\mathrm{e}^{[15,19]}$.

\section{MECHANISMS UNDERLYING ACUTE HYPERTENSIVE RESPONSE RELATED INJURY}

How does acute hypertensive response increase the hematoma expansion? Probably one of the most probable explanation is that higher systemic BP causes a predisposition to continued intraparenchymal bleeding and perihematomal edema by transmitting higher hydrostatic pressure to damaged small arteries. Furthermore, the fact that treatment with hemostatic agents such as intravenous (IV) recombinant activated Factor VII (rfVIIa) reduces the hematoma expansion rate suggests that there may be additional interaction between high SBP and the hemostatic system ${ }^{[20]}$. It has been shown that the renin angiotensin system (RAS), which is a peptide hormone system involved in BP regulation and blood volume homeostasis in the circulation with increased activity in the hypertensive state, acts as a local paracrine system in the brain ${ }^{[21]}$. The systemic and tissue RAS activity increases in hypertensive state ${ }^{[22]}$. Angiotensin II (Ang II), a powerful vasoconstrictor regulated by RAS that increases $\mathrm{BP}$ and cerebral blood flow (CBF), increases venous thrombus formation and exert prothrombotic activity in an experimental model of arterial thrombosis, possibly due to the fibrinolysis inhibition ${ }^{[22,23]}$. Despite the increased level of Ang II with prothrombotic effect, the question of how the hematoma size increases in patients with hypertensive ICH is waiting to be answered. Increased BP and increased CBF due to the effect of Ang II may impede the hemostasis ${ }^{[12]}$. Ang II-mediated oxidative stress and activation of matrix metalloproteases in the cerebral vessels in the hemorrhage area may also contribute to hematoma expansion ${ }^{[24,25]}$. 
In addition to aggravating primary brain injury by causing increased hematoma, elevated BP also influences secondary brain injury through affecting brain swelling, inflammation, apoptosis and necrosis. RAS inhibition has been reported to reduce inflammation, oxidative stress, and infarct volume, modulate nitric oxide synthase isoenzymes in animal stroke models, and improve functional outcome in stroke patients ${ }^{[12]}$. Stimulation of brain and cerebrovascular Ang II systems in rats has been shown to increase micro vessel permeability, and expression of proinflammatory factors and contribute to vasoconstriction ${ }^{[26]}$. Angiotensin II type 1 receptors (Ang II AT1) in neurons, astrocytes, microglia, and brain microvascular endothelial cells are likely to play a role in inflammatory response modulation in hypertensive state ${ }^{[27-29]}$. Long-term brain Ang II AT1 receptor blockade protects the brain from the pathological effects of stress and from ischemia, partly through peripheral anti-inflammatory effects ${ }^{[30]}$. Evidence suggests that elevated systemic BP can increase norepinephrine concentration in the subfornix ${ }^{[31]}$. Norepinephrine acts at beta-adrenoreceptors and selective beta-adrenoreceptor 1 antagonists have been shown to provide neuroprotective effects in ischemic stroke models ${ }^{[32,33]}$.

\section{RECONSIDERING THE DEFINITION OF ACUTE HYPERTENSIVE RESPONSE}

In a rat model of collagenase-induced ICH study, an ultra-acute elevation in SBP following induction of ICH has been observed in both normotensive rats and rats with renovascular hypertension (RVHT) [mean arterial pressure (MAP) in RVHT rats increased from $131.9 \pm 12.6 \mathrm{mmHg}$ to $138.9 \mathrm{mmHg}$; in normotensive rats increased from $85.7 \pm 11.5 \mathrm{mmHg}$ to $89.5 \mathrm{mmHg}$ at $30 \mathrm{~min}$ after $\mathrm{ICH}$ ] and elevated SBP increased hematoma volume, brain edema, and perihematomal apoptosis ${ }^{[12]}$. However, the hematoma volume was $60 \%$ greater in RVHT rats and correlated with more severe neurological deficits at three weeks. Additionally, the increase in heart rate observed during the hyperacute phase in normotensive rats was not observed in RVHT rats, probably due to an altered cardiovascular response.

In another experimental study with rodents, researchers compared the amount of bleeding that chronic hypertensive and normotensive rats experienced after brain surgery while the BP values of the subjects are kept at the presurgical levels ${ }^{[34]}$. Comparison of normotensive, acutely hypertensive, spontaneously hypertensive (antihypertensive treatment given and not given) rats showed that only the hematoma volume of acutely hypertensive rats was larger. These findings were thought to provide evidence that the brains of spontaneously hypertensive rats are presumably protected against excessive bleeding due to increased resistance in large cerebral arteries resulting in reduced cerebral intravascular pressure.

Acute hypertensive response is defined as "SBP $\geq 140 \mathrm{mmHg}$ " consistent with the 2003 World Health Organization/International Society of Hypertension statement but, is it appropriate to use this definition in both patients with and without chronic hypertension ${ }^{[35]}$ ? Should the diagnosis and treatment criteria for acute hypertensive response be the same in two patients with ICH of the same size and location, one with chronic hypertension and the other without, represented by the same arterial BP value or the same amount of increase in pre-morbid BP values? From the animal experiments mentioned above, we can easily deduce that the outcome of these two patients will be different even with the same treatment. However, it seems quite difficult to classify patients in this way in randomized studies. Since hypertension can be asymptomatic for years, it is nearly impossible to predict whether the hypertension detected in an $\mathrm{ICH}$ patient at the time of presentation without a history of chronic hypertension is the cause, the result or a contributing factor, except for a few clues (patients over 45 years of age; the observation of left ventricular hypertrophy by electrocardiogram, and cardiomegaly by chest radiography; retinal changes; the observation of homogeneous, oval or round smooth-looking hematomas, surrounded by a thin rim of edema that can expand for a few days, with periventricular white matter and the protuberance involvement in neuroimaging suggest that the ICH is secondary to hypertension-related angiopathy $)^{[16,36]}$. However, 
considering that the most common cause of ICH is chronic hypertension, we can infer that previous studies mostly examined patients who developed ICH on the basis of chronic hypertension.

Apart from not considering history of previous hypertension, there is also the problem that the definition "SBP $\geq 140 \mathrm{mmHg}$ " does not consider the relationship between the degree of pretreatment BP and outcome. Studies showed that the relationship between acute hypertensive response and hematoma enlargement and mortality is evident at higher SBPs. In the retrospective study by Dandapani et al ${ }^{[37]}$, patients with SBP > $196 \mathrm{mmHg}$ on admission had a higher mortality rate than those with SBP $\leq 196 \mathrm{mmHg}(44 \%$ vs. $18 \%)$. Similarly, according to the study by Kazui et al. ${ }^{[19]}, \mathrm{SBP} \geq 200 \mathrm{mmHg}$ on admission independently predisposed to hematoma expansion. In the Intensive Blood Pressure Reduction in Acute Cerebral Haemorrhage trial (INTERACT), the most significant attenuation of hematoma expansion with early intensive BP treatment was observed in patients with $\mathrm{SBP} \geq 181 \mathrm{mmHg}$ on admission ${ }^{[38]}$. Thus, the definition "a SBP of $\geq 140 \mathrm{mmHg}$ " may provide a uniform standard for measuring prevalence but does not define therapeutic thresholds for antihypertensive treatment ${ }^{[39,40]}$.

\section{MANAGEMENT OF ACUTE HYPERTENSIVE RESPONSE}

Reducing increased BP following an $\mathrm{ICH}$, which has been found to be associated with hematoma enlargement, perihematomal edema, and poor outcomes, has been recognized as a potential strategy ${ }^{[17]}$. On the other hand, due to the possibility that acute hypertension might be a protective response to increased ICP, there were concerns about the potential risk of worsening perihaematomal ischaemia from aggressive early BP reduction especially in the setting of chronic hypertension, which raises the lower limit of the CBF autoregulation curve ${ }^{[41]}$. U- or J-shaped associations between BP levels and poor outcome have been demonstrated for ischemic stroke ${ }^{[42]}$. For ICH, the results were variable in different studies. A prospective study reported higher mortality rates in patients with $\mathrm{SBP}>220 \mathrm{mmHg}$ or $<120 \mathrm{mmHg}$ compared with patients with SBP 141-160 $\mathrm{mmHg}^{[43]}$. In a retrospective chart review with 105 spontaneous ICH patients, rapid MAP decline within the first $24 \mathrm{~h}$ was associated with increased mortality ${ }^{[44]}$. However, in a brain oxygenation positron emission tomography study with 19 acute ICH patients, researchers found no evidence for ischemia in the periclot zone of hypoperfusion 5 to $22 \mathrm{~h}$ after the onset of bleeding ${ }^{[45]}$. Studies suggested that reduced metabolism (hibernation) and preserved autoregulation in the perihematoma region led to the tolerance of BP lowering ${ }^{[46,47]}$. In large phase III, multicentre, prospective, randomised, controlled trials, lowering of SBP to $<140 \mathrm{mmHg}$ in the acute phase has been found saf $\mathrm{e}^{[48,49]}$. However, the effect of BP lowering for special groups continues to be investigated in clinical trials. Antihypertensive Treatments for Spontaneous Intracerebral Hemorrhage in Patients with Cerebrovascular Stenosis (ATICHST) is a recent clinical trial designed to investigate the effects of intensive $\mathrm{BP}$ reduction $(<140 \mathrm{mmHg})$ in spontaneous ICH patients with cerebrovascular stenosis (constituting $20 \%$ to $54 \%$ of ICH patients) ${ }^{[50]}$. Patients with $30 \%-70 \%$ stenosis in the anterior or posterior circulation in computed tomography (CT) angiography and with a SBP between 150-220 mmHg will be included. With this ongoing study, it is aimed to understand whether intensive SBP lowering in the presence of cerebrovascular stenosis increases the risk of ischemia and infarction.

\section{CURRENT GUIDELINE RECOMMENDATIONS FOR ELEVATED BP IN SPONTANEOUS ICH}

The main issues related to the management of acute hypertensive response can be considered to consist of treatment threshold, and time window, type of antihypertensive drug and routes of administration, and target BP. Which of these do the current guidelines clarify? 
American Heart Association/American Stroke Association (AHA/ASA) made its last guidance update on spontaneous ICH in 2015. There were concerns that acute BP lowering may worsen cerebral injury based on the idea that ischaemic penumbra in the vicinity of hematoma may progress to permanent cerebral injury if $\mathrm{BP}$ is lowered quickly and too much but studies indicated that BP reduction decreases hematoma expansion without affecting perihematomal blood flow ${ }^{[1-54]}$. Also, although it was argued for heterogeneity of used antihypertensive drugs, the INTERACT-2 showed that aggressive (target $\mathrm{SBP}<140 \mathrm{mmHg}$ within $1 \mathrm{~h}$ ) BP lowering is feasible and safe and, moreover, it can be effective for improving better functional outcome compared with conservative BP lowering ${ }^{[49]}$. Therefore, unlike previous guidelines that were hesitant and cautious in aggressive BP lowering, the 2015 guidelines recommended acute lowering of SBP to $140 \mathrm{mmHg}$ for patients presenting with SBP between $150-220 \mathrm{mmHg}$ and without contraindication to acute BP treatment (class I; level of evidence A) and considering aggressive reduction of BP with a continuous IV infusion and frequent BP monitoring for those presenting with SBP $>220 \mathrm{mmHg}$ (class IIb; level of evidence $\mathrm{C}$ ). However, there were no specific recommendations regarding the treatment window and the type of antihypertensive agent and route of administration ${ }^{[39]}$.

According to European Stroke Initiative's 2006 recommendations, the necessary BP target was different for those with and without previous history of hypertension and suggestions were hesitant and cautious in aggressive BP lowering as a hypertensive patient may not be able to maintain cerebral perfusion at a significantly lower SBP. If the patient has a history of hypertension and SBP $>180 \mathrm{mmHg}$ or DBP $>$ $105 \mathrm{mmHg}$, it was recommended to reduce $\mathrm{BP}$ to $<170 / 100 \mathrm{mmHg}$ or MAP to $<125 \mathrm{mmHg}$, avoiding lowering pressure by more than $20 \%$ of MAP on admission (class IV; level C). For patients with unknown history of hypertension, if $\mathrm{SBP}>160 \mathrm{mmHg}$ or $\mathrm{DBP}>95 \mathrm{mmHg}$, it was recommended to reduce $\mathrm{BP}$ to $<$ $150 / 90 \mathrm{mmHg}$ or MAP to $<100 \mathrm{mmHg}$, avoiding lowering pressure by more than $20 \%$ of MAP on admission (class IV; level C). It was also recommended to adapt thresholds to cerebral perfusion pressure > $70 \mathrm{mmHg}$ (class IV; level C) and to use IV drugs for better control (good clinical practice) ${ }^{[55]}$. These recommendations were updated by European Stroke Organisation in 2014. Updated guidelines recommended intensive BP reduction (target $\mathrm{SBP}<140 \mathrm{mmHg}$ in $<1 \mathrm{~h}$ ) in ICH within $6 \mathrm{~h}$ of onset without recommending a specific agent, noting that it is safe and may be superior to a SBP target of $<180 \mathrm{mmHg}$ (quality of evidence moderate, strength of recommendation weak). No recommendation has been made regarding the lower limit of $\mathrm{BP}^{[40]}$. At the time of writing this article, "Management of Hypertension in Acute Ischemic Stroke and Intracerebral Hemorrhage" guideline was in development.

\section{BLOOD PRESSURE REDUCTION IN ACUTE ICH IN RANDOMIZED TRIALS AND META- ANALYSIS}

The debate about whether to treat hypertension in acute stroke patients started 36 years ago ${ }^{[56]}$. After 1997, the relationship between acute hypertensive response and hematoma expansion, which was not recommended to be treated due to perihematomal ischemia concerns, began to be elucidated, and moderate lowering of BP was recommended based on the case series results ${ }^{[57]}$. Since 2004, the effects of aggressive BP lowering on hematoma expansion and patient outcomes have continued to be investigated. Although we have some information about the safety of treatment of acute hypertensive response at the point we have reached in 36 years, we can state that our knowledge about the benefits of treatment, timing of treatment, medications that should be used, the treatment threshold and target BP is still insufficient. Randomized clinical trials (see Table 1) with variable antihypertensive agents, different theoropathic windows and thresholds, different sample sizes and different time frames to achieve target BP, and related subgroup analyzes and meta-analyzes make it difficult to reach a definite conclusion. 
Table 1. Summary of randomized clinical trials evaluating treatment of acute hypertensive response in patients with intracerebral hemorrhage

\begin{tabular}{|c|c|c|c|c|c|c|c|c|c|c|c|c|c|c|c|c|c|}
\hline \multirow{2}{*}{$\begin{array}{l}\text { Opinion } \\
\text { on early } \\
\text { intensiv } \\
\text { e } \\
\text { antihper } \\
\text { tensive } \\
\text { therapy } \\
\text { accordin } \\
\text { g to } \\
\text { study } \\
\text { results }\end{array}$} & \multirow[b]{2}{*}{ Trial } & \multirow[b]{2}{*}{ Patients } & \multirow[b]{2}{*}{$\begin{array}{l}\text { Eligibilit } \\
\text { y BP } \\
\text { (mmHg) }\end{array}$} & \multirow[b]{2}{*}{$\begin{array}{l}\text { Time } \\
\text { frame } \\
\text { for } \\
\text { treatme } \\
\text { nt }\end{array}$} & \multirow[b]{2}{*}{$\begin{array}{l}\text { Antihyp } \\
\text { ertensiv } \\
\text { e drugs }\end{array}$} & \multirow[b]{2}{*}{$\begin{array}{l}\text { Follow- } \\
\text { up time }\end{array}$} & \multicolumn{4}{|c|}{ Intensive BP lowering group } & \multicolumn{4}{|c|}{ Conservative BP lowering } & \multirow[b]{2}{*}{$\begin{array}{l}\text { Primary } \\
\text { outcome }\end{array}$} & \multirow[b]{2}{*}{$\begin{array}{l}\text { Outcom } \\
\text { e } \\
\text { intensiv } \\
\text { e/active } \\
\text { treatme } \\
\text { nt group }\end{array}$} & \multirow[b]{2}{*}{$\begin{array}{l}\text { Outcom } \\
\text { e } \\
\text { standard } \\
\text { /control } \\
\text { group }\end{array}$} \\
\hline & & & & & & & $\begin{array}{l}\text { Number } \\
\text { of } \\
\text { patients } \\
\text { (ICH) }\end{array}$ & $\begin{array}{l}\text { Target } \\
\text { BP } \\
\text { (mmHg) }\end{array}$ & $\begin{array}{l}\text { Time } \\
\text { from } \\
\text { onset to } \\
\text { treatme } \\
\text { nt }\end{array}$ & $\begin{array}{l}\text { Time to } \\
\text { achieve } \\
\text { targeted } \\
\text { BP }\end{array}$ & $\begin{array}{l}\text { Number } \\
\text { of } \\
\text { patients } \\
\text { (ICH) }\end{array}$ & $\begin{array}{l}\text { Target } \\
\text { BP } \\
(\mathrm{mmHg})\end{array}$ & $\begin{array}{l}\text { Time } \\
\text { from } \\
\text { onset to } \\
\text { treatme } \\
\text { nt }\end{array}$ & $\begin{array}{l}\text { Time to } \\
\text { achieve } \\
\text { targeted } \\
\text { BP }\end{array}$ & & & \\
\hline $\begin{array}{l}\text { Supporte } \\
\text { d }\end{array}$ & $\begin{array}{l}\text { INTERAC } \\
T^{[38]} \\
2008\end{array}$ & $\begin{array}{l}\mathrm{ICH} \\
\text { patients }\end{array}$ & $\begin{array}{l}\text { SBP 150- } \\
220\end{array}$ & $6 \mathrm{~h}$ & $\begin{array}{l}\text { Urapidil, } \\
\text { labetalol, } \\
\text { phentola } \\
\text { mine, } \\
\text { nicardipin } \\
\text { e, nitrates } \\
\text { (IV and } \\
\text { patch), } \\
\text { hydralazin } \\
\text { e, } \\
\text { metoprolo } \\
\text { I, diuretics }\end{array}$ & 90 days & 203 & $<140$ & $240 \min$ & $\begin{array}{l}\text { Within } 1 \mathrm{~h} \\
\text { in } 42 \% \text { of } \\
\text { patients } \\
\text { and within } \\
6 \mathrm{~h} 66 \% \\
\text { of } \\
\text { patients }\end{array}$ & 201 & $<180$ & $280 \mathrm{~min}$ & - & $\begin{array}{l}\text { Proportio } \\
\text { nal } \\
\text { change in } \\
\text { hematom } \\
\text { a volume } \\
\text { at } 24 \mathrm{~h}\end{array}$ & $\begin{array}{l}\text { Mean } \\
\text { proportio } \\
\text { nal } \\
\text { haemato } \\
\text { ma } \\
\text { growth } \\
\text { was 13.7\% }\end{array}$ & $\begin{array}{l}\text { Mean } \\
\text { proportio } \\
\text { nal } \\
\text { haemato } \\
\text { ma } \\
\text { growth } \\
\text { was } \\
36.3 \%\end{array}$ \\
\hline Neutral & $\begin{array}{l}\text { Koch et al. } \\
{[52], 2008}\end{array}$ & $\begin{array}{l}\mathrm{ICH} \\
\text { patients }\end{array}$ & $\begin{array}{l}\text { MAP } \geq \\
110\end{array}$ & $8 \mathrm{~h}$ & $\begin{array}{l}\text { Labetalol, } \\
\text { nicardipin } \\
\text { e, sodium } \\
\text { nitropruss } \\
\text { ide }\end{array}$ & 90 days & 21 & $\mathrm{MAP}<110$ & $3.2 \pm 2.2 \mathrm{~h}$ & $\begin{array}{l}163.5 \pm \\
163.8 \mathrm{~min}\end{array}$ & 21 & $\begin{array}{l}\text { MAP 110- } \\
130\end{array}$ & $3.2 \pm 2.2 \mathrm{~h}$ & $\begin{array}{l}87.1 \pm \\
59.6 \text { min }\end{array}$ & $\begin{array}{l}\text { Clinical } \\
\text { deteriorati } \\
\text { on (NIHSS } \\
\text { score } \\
\text { drop } \geq 2 \\
\text { points) } \\
\text { within the } \\
\text { first } 48 \mathrm{~h} \text {. } \\
\text { Hematom } \\
\text { a } \\
\text { enlargeme } \\
\text { nt at } 24 \mathrm{~h} \\
\text { was a } \\
\text { secondary } \\
\text { endpoint }\end{array}$ & $\begin{array}{l}11.9 \pm 29.7 \\
\text { percent } \\
\text { average } \\
\text { increase } \\
\text { in ICH } \\
\text { volume } \\
\text { and } 25.7 \pm \\
45.5 \text { in } \\
\text { edema } \\
\text { volume, } \\
\text { no } \\
\text { significant } \\
\text { difference } \\
\text { s in early } \\
\text { neurologic } \\
\text { al } \\
\text { deteriorati } \\
\text { on and } \\
\text { clinical } \\
\text { outcomes } \\
\text { at } 90 \text { days }\end{array}$ & $\begin{array}{l}18.2 \pm \\
46.8 \\
\text { percent } \\
\text { average } \\
\text { increase } \\
\text { in ICH } \\
\text { volume } \\
\text { and } 35.7 \pm \\
48.7 \text { in } \\
\text { edema } \\
\text { volume, } \\
\text { no } \\
\text { significant } \\
\text { difference } \\
\text { s in early } \\
\text { neurologic } \\
\text { al } \\
\text { deteriorati } \\
\text { on and } \\
\text { clinical } \\
\text { outcomes } \\
\text { at } 90 \text { days }\end{array}$ \\
\hline
\end{tabular}




\begin{tabular}{|c|c|c|c|c|c|c|c|c|c|c|c|c|c|c|c|c|c|}
\hline Neutral & $\begin{array}{l}\text { ICH } \\
\text { ADAPT }^{[59} \\
]_{1} \\
2013\end{array}$ & $\begin{array}{l}\mathrm{ICH} \\
\text { patients }\end{array}$ & $\mathrm{SBP}>150$ & $24 \mathrm{~h}$ & $\begin{array}{l}\text { Labetalol, } \\
\text { hydralazin } \\
\text { e, } \\
\text { enalapril }\end{array}$ & 90 days & 39 & $<150$ & $\begin{array}{l}7.83 \\
(3.25- \\
16.75) \mathrm{h}\end{array}$ & $\begin{array}{l}\text { Within } 2 \mathrm{~h} \\
\text { in } 79 \% \text { of } \\
\text { patients }\end{array}$ & 36 & $<180$ & $\begin{array}{l}8.54(3.8- \\
15.75) h\end{array}$ & $\begin{array}{l}\text { Within } 2 \mathrm{~h} \\
\text { in } 100 \% \\
\text { of } \\
\text { patients }\end{array}$ & $\begin{array}{l}\text { Perihemat } \\
\text { oma } \\
\text { relative } \\
\text { CBF }\end{array}$ & $\begin{array}{l}\text { Perihemat } \\
\text { oma } \\
\text { relative } \\
\text { CBF } 0.86 \\
\pm 0.12\end{array}$ & $\begin{array}{l}\text { Perihemat } \\
\text { oma } \\
\text { relative } \\
\text { CBF } 0.89 \\
\pm 0.09\end{array}$ \\
\hline $\begin{array}{l}\text { Supporte } \\
\text { d }\end{array}$ & $\begin{array}{l}\text { INTERAC } \\
\text { T-2 }^{[49]} \\
2013\end{array}$ & $\begin{array}{l}\mathrm{ICH} \\
\text { patients }\end{array}$ & $\begin{array}{l}\text { SBP 150- } \\
220\end{array}$ & $6 \mathrm{~h}$ & $\begin{array}{l}\text { Labetalol, } \\
\text { metoprolo } \\
\text { l, } \\
\text { hydralazin } \\
e_{\text {, }} \\
\text { phentola } \\
\text { mine, } \\
\text { nicardipin } \\
e_{\text {, }} \\
\text { nimodipin } \\
e_{,} \\
\text {nitroglyce } \\
\text { rin, } \\
\text { urapidil, } \\
\text { esmolol, } \\
\text { clonidine, } \\
\text { enalapril, } \\
\text { nitropruss } \\
\text { ide, } \\
\text { furosemid } \\
\text { e, and oral } \\
\text { agents }\end{array}$ & 90 days & 1399 & $<140$ & $\begin{array}{l}4(2.9- \\
5.1) h\end{array}$ & $\begin{array}{l}\text { Within 1h } \\
\text { in } 33.4 \% \\
\text { of } \\
\text { patients }\end{array}$ & 1430 & $<180$ & $\begin{array}{l}4.5(3-7) \\
h\end{array}$ & - & $\begin{array}{l}\text { Death or } \\
\text { disability } \\
\text { by } m R S \\
(3-6) \text { at } \\
\text { three } \\
\text { months } \\
\text { post } \\
\text { randomiza } \\
\text { tion }\end{array}$ & $\begin{array}{l}52 \% \text { had } \\
\text { a poor } \\
\text { outcome } \\
\text { event }\end{array}$ & $\begin{array}{l}55.6 \% \\
\text { had a } \\
\text { poor } \\
\text { outcome } \\
\text { event }\end{array}$ \\
\hline $\begin{array}{l}\text { Supporte } \\
\text { d }\end{array}$ & $\begin{array}{l}\text { Gong et al. } \\
[66,6]] \\
2015\end{array}$ & $\begin{array}{l}\mathrm{ICH} \\
\text { patients }\end{array}$ & $\mathrm{SBP}>160$ & $4 \mathrm{~h}$ & $\begin{array}{l}\text { Nitroglyce } \\
\text { rin }\end{array}$ & 14 days & 60 & $130-140$ & $\begin{array}{l}2.24 \pm \\
1.23\end{array}$ & $1 \mathrm{~h}$ & 60 & $160-180$ & $\begin{array}{l}2.13 \pm 1.05 \\
h\end{array}$ & $1 \mathrm{~h}$ & $\begin{array}{l}\text { Hematom } \\
\text { a } \\
\text { expansion } \\
\text {, edema } \\
\text { volume, } \\
\text { serum } \\
\text { MMP-9 } \\
\text { level }\end{array}$ & $\begin{array}{l}\text { Hematom } \\
\text { a } \\
\text { expansion } \\
: 5 \\
(8.33 \%) \\
\text { NIHSS } \\
\text { score: } \\
6.28 \pm \\
3.68 \\
\text { Lower } \\
\text { edema } \\
\text { volume } \\
\text { and serum } \\
\text { MMP-9 } \\
\text { levels } \\
\text { after } 5 \\
\text { and } 14 \\
\text { days }\end{array}$ & $\begin{array}{l}\text { Hematom } \\
\text { a } \\
\text { expansion } \\
: 14 \\
(23.33 \%) \\
\text { NIHSS } \\
\text { score: } \\
7.82 \pm \\
4.28 \\
\text { Higher } \\
\text { edema } \\
\text { volume } \\
\text { and serum } \\
\text { MTP-9 } \\
\text { levels } \\
\text { after } 5 \\
\text { and } 14 \\
\text { days }\end{array}$ \\
\hline $\begin{array}{l}\text { Arguing/ } \\
\text { against }\end{array}$ & $\begin{array}{l}\text { ATACH- } \\
2^{[48,68]} \\
2016\end{array}$ & $\begin{array}{l}\text { ICH } \\
\text { patients }\end{array}$ & $\begin{array}{l}\text { SBP } 180- \\
240\end{array}$ & $4.5 \mathrm{~h}$ & $\begin{array}{l}\text { Nicardipin } \\
\text { e, } \\
\text { labetalol, }\end{array}$ & 90 days & 500 & 110-139 & $\begin{array}{l}182.2 \pm \\
57.2 \mathrm{~min}\end{array}$ & $\begin{array}{l}\text { Within } 2 \mathrm{~h} \\
\text { in } 87.8 \% \\
\text { of }\end{array}$ & 500 & 140-179 & $\begin{array}{l}184.7 \pm \\
56.7 \mathrm{~min}\end{array}$ & $\begin{array}{l}\text { Within } 2 \mathrm{~h} \\
\text { in } 99.2 \% \\
\text { of }\end{array}$ & $\begin{array}{l}\text { Death or } \\
\text { disability } \\
\text { by mRS }\end{array}$ & $\begin{array}{l}38.7 \% \\
\text { had a } \\
\text { primary }\end{array}$ & $\begin{array}{l}37.7 \% \\
\text { had a } \\
\text { primary }\end{array}$ \\
\hline
\end{tabular}




\begin{tabular}{|c|c|c|c|c|c|c|c|c|c|c|}
\hline & & & & & $\begin{array}{l}\text { diltiazem, } \\
\text { uradipil }\end{array}$ & & patients & $\begin{array}{l}(4-6) \text { at } \\
\text { three } \\
\text { months } \\
\text { post } \\
\text { randomiza } \\
\text { tion }\end{array}$ & $\begin{array}{l}\text { outcome } \\
\text { event }\end{array}$ & $\begin{array}{l}\text { outcome } \\
\text { event }\end{array}$ \\
\hline Neutral & $\begin{array}{l}\underset{[54,58],}{\text { ATACH }} \\
2010^{\prime}\end{array}$ & $\begin{array}{l}\text { ICH } \\
\text { patients }\end{array}$ & $S B P \geq 170$ & $6 \mathrm{~h}$ & $\begin{array}{l}\text { Nicardipin } \\
\mathrm{e}\end{array}$ & $\begin{array}{l}90 \pm 15 \\
\text { days }\end{array}$ & $\begin{array}{l}\text { Cohort 1: } 18 \text { patients, target SBP of } 170-200 \mathrm{mmHg} \\
\text { Cohort 2: } 20 \text { patients, target SBP of } 140-170 \mathrm{mmHg} \\
\text { Cohort 3: } 22 \text { patients, target SBP of } 110-140 \mathrm{mmHg} \\
\text { Mean time from symtom onset to initiating treatment was } 3.94 \mathrm{~h}, 4.13 \mathrm{~h} \text { and } 4.44 \mathrm{~h} \text {, } \\
\text { respectively and the goals were achieved in } 90 \% \text { of the patients by } 2 \mathrm{~h}\end{array}$ & $\begin{array}{l}\text { Treatment } \\
\text { failure, } \\
\text { neurologic } \\
\text { deteriorati } \\
\text { ons within } \\
24 \mathrm{~h} \text {, and } \\
\mathrm{SAE} \\
\text { within } 72 \\
\mathrm{~h}\end{array}$ & $\begin{array}{l}\text { Rates } \\
\text { were } 21 \% \\
\text { for } \\
\text { hematom } \\
\text { a } \\
\text { expansion } \\
\text { and } 35 \% \\
\text { for poor } \\
\text { three- } \\
\text { month } \\
\text { outcome }\end{array}$ & $\begin{array}{l}\text { Rates } \\
\text { were } 31 \% \\
\text { for } \\
\text { hematom } \\
\text { a } \\
\text { expansion } \\
\text { and } 48 \% \\
\text { for poor } \\
\text { three- } \\
\text { month } \\
\text { outcome }\end{array}$ \\
\hline $\begin{array}{l}\text { Neutral } \\
\text { but, } \\
\text { secondar } \\
\text { y analysis } \\
\text { supporte } \\
\text { d }\end{array}$ & $\begin{array}{l}\text { ENOS } \\
{[64,65]^{\prime}} \\
2015\end{array}$ & $\begin{array}{l}\text { Ischemic } \\
\text { or } \\
\text { hemorrha } \\
\text { gic stroke } \\
\text { patients }\end{array}$ & $\begin{array}{l}\text { SBP 140- } \\
220\end{array}$ & $48 \mathrm{~h}$ & $\begin{array}{l}\text { Transder } \\
\text { mal } \\
\text { glyceryl } \\
\text { trinitrate }\end{array}$ & 90 days & $\begin{array}{l}\text { GTN group: } 310 \text { patients } \\
\text { No GTN group: } 319 \text { patients } \\
\text { Time from onset to treatment was } 25.2 \pm 13.1 \mathrm{~h} \text { in the GTN group, } 25.1 \pm 12.9 \mathrm{~h} \text { in the no GTN } \\
\text { group } \\
\text { Baseline BP was } 172 / 93 \mathrm{mmHg} \text { and significantly lower in GTN group }(-7.5 /-4.2 \mathrm{mmHg}) \text { on } \\
\text { day } 1\end{array}$ & $\begin{array}{l}\text { Mortality } \\
\text { rate and } \\
\text { mRS at } 3 \\
\text { months } \\
\text { post } \\
\text { randomiza } \\
\text { tion }\end{array}$ & $\begin{array}{l}\text { Median } \\
\text { mRS } \\
\text { grade of } 3 \\
\text { (IQR 2) }\end{array}$ & $\begin{array}{l}\text { Median } \\
\text { mRS } \\
\text { grade of } 3 \\
\text { (IQR 2) }\end{array}$ \\
\hline $\begin{array}{l}\text { Arguing/ } \\
\text { against }\end{array}$ & $\begin{array}{l}\text { RIGHT- } \\
2^{[73]}, 2019\end{array}$ & $\begin{array}{l}\text { Ischemic } \\
\text { or } \\
\text { hemorrha } \\
\text { gic stroke } \\
\text { patients }\end{array}$ & $S B P \geq 120$ & $4 \mathrm{~h}$ & $\begin{array}{l}\text { Transder } \\
\text { mal } \\
\text { glyceryl } \\
\text { trinitrate }\end{array}$ & 90 days & $\begin{array}{l}\text { GTN group: } 74 \text { patients } \\
\text { Sham group: } 71 \text { patients } \\
\text { Median SBP was } 176 \pm 27 \mathrm{mmHg} \text {, mean time from onset to treatment was } 74(45-110) \text { min, } \\
\text { BP was lower in the GTN group by admission to hospital (mean, }-4.4 /-3.5 \mathrm{mmHg} \text { ) }\end{array}$ & $\begin{array}{l}\mathrm{mRS} \text { at } 90 \\
\text { days post } \\
\text { randomiza } \\
\text { tion }\end{array}$ & $\begin{array}{l}\text { Mean } \\
\text { mRS } \\
\text { grade of } \\
4.9( \pm 1.4)\end{array}$ & $\begin{array}{l}\text { Mean } \\
\text { mRS } \\
\text { grade of } \\
4.3( \pm 1.6)\end{array}$ \\
\hline
\end{tabular}

ATACH: Antihypertensive Treatment of Acute Cerebral Hemorrhage; BP: blood pressure; CBF: cerebral blood flow; CT: computerized tomography; ENOS: efficacy of nitric oxide in stroke; ICH: intracerebral hemorrhage; ICH ADAPT: intracerebral hemorrhage acutely decreasing arterial pressure trial; INTERACT: Intensive Blood Pressure Reduction in Acute Cerebral Haemorrhage Trial; IQR: interquartile range; MAP: mean arterial pressure; MMP-9: matrix metalloproteinase-9; mRS: modified Rankin scale; NIHSS: National Institutes of Health Stroke Scale; RIGHT: Rapid Intervention with Glyceryl Trinitrate in Hypertensive Stroke Trial; SAE: serious adverse event; SBP: systolic blood pressure.

\section{Koch et al. ${ }^{[52]}, 2008$}

Koch et al.$^{[52]}$ investigated the feasibility and safety of aggressive BP lowering in this prospective, randomized, single-center study. They used MAP instead of SBP in line with AHA guidelines at the time of the study. Forty-two patients with ICH, within $8 \mathrm{~h}$ of onset, with elevated MAP ( $\geq 110 \mathrm{mmHg}$ ) were randomized to standard treatment (target MAP 110-130 $\mathrm{mmHg}, n=21$ ) or aggressive treatment (target $\mathrm{MAP}<110 \mathrm{mmHg}$ ). Baseline characteristics were similar except that the ICH and edema volumes were larger in the aggressive BP group. IV labetalol, nicardipine, or sodium nitroprussid was used and target BP was obtained within $87.1 \pm 59.6 \mathrm{~min}$ in the standard treatment group and $163.5 \pm 163.8 \mathrm{~min}$ in the aggressive BP treatment group. No significant differences were found between two groups in terms of hematoma and edema growth, early neurological deterioration, and clinical outcome at 90 days. 


\section{INTERACT, 2008}

This pilot trial compared the effect of BP lowering on haematoma expansion ${ }^{[38]}$. Four hundred and four ICH patients with high SBP (150-220 mmHg) were randomized to an intensive treatment group (target SBP < $140 \mathrm{mmHg} ; n=203$ ) and a guideline-based BP treatment group (target SBP $<180 \mathrm{mmHg} ; n=201$ ) within $6 \mathrm{~h}$ of symptom onset. The lower limit of SBP required for discontinuation of antihypertensive treatment was $130 \mathrm{mmHg}$. Variable antihypertensive agents were used in the study. In the intensive treatment group, target SBP of $140 \mathrm{mmHg}$ was achieved in $42 \%$ of patients within $1 \mathrm{~h}$ and $66 \%$ of patients within $6 \mathrm{~h}$. Intensive BP reduction resulted in less increase in hematoma volume (absolute risk reduction: $8 \%$ ) and was found clinically feasible and well tolerated.

\section{ATACH, 2010}

In the Antihypertensive Treatment of Acute Cerebral Hemorrhage (ATACH) trial treatment failure, early neurologic deterioration, and serious adverse event (SAE) within $72 \mathrm{~h}$ of the groups with target SBP of 170$200 \mathrm{mmHg}, 140-170 \mathrm{mmHg}$ and 110-140 $\mathrm{mmHg}$ were investigated ${ }^{[58]}$. Sixty ICH patients, within first $6 \mathrm{~h}$ after symptom onset, with SBP $\geq 170 \mathrm{mmHg}$ were included and IV nicardipine was used. The average duration from symptom onset to start of treatment was $3.94 \mathrm{~h}, 4.13 \mathrm{~h}$ and $4.44 \mathrm{~h}$, respectively. In $90 \%$ of patients, SBP targets were achieved within $2 \mathrm{~h}$. Neurological deterioration and SAE rates were lower than expected in each group according to pre-determined thresholds. The magnitude of SBP reduction was associated with attenuation of hematoma enlargement and poor three-month outcomes (the rates were $21 \%$ vs. $31 \%$ for hematoma enlargement, and $35 \%$ vs. $48 \%$ for poor three-month outcomes with SBP reduction of $>54 \mathrm{mmHg}$ compared with $\leq 54 \mathrm{mmHg}$ at $2 \mathrm{~h})^{[54]}$.

\section{ICH ADAPT, 2013}

The Intracerebral Hemorrhage Acutely Decreasing Arterial Pressure Trial (ICH ADAPT) tested whether BP reduction in acute ICH patients affected CBF. ICH patients within $24 \mathrm{~h}$ of symptom onset, with SBP > $150 \mathrm{mmHg}$ were randomized to a treatment group targeting a SBP of $<150 \mathrm{mmHg}(n=39)$ or $<180 \mathrm{mmHg}$ $(n=36)$. Labetalol, hydralazine and enalapril were used. Despite similar initial SBP, mean SBP after $2 \mathrm{~h}$ was significantly lower in the intensive treatment group (140 $\pm 19 \mathrm{mmHg} v s .162 \pm 12 \mathrm{mmHg}$ ). Perihematomal relative CBF was similar in both groups. Although the study was underpowered to compare these events, ICH volume growth, mortality, and disability scores were all similar between two groups ${ }^{[59]}$.

\section{INTERACT-2, 2013}

In the INTERACT-2 trial, ICH patients with SBP 150-220 mmHg were randomized to intensive BP treatment (target $\mathrm{SBP}<140 \mathrm{mmHg} ; n=1399$ ) or conservative $\mathrm{BP}$ treatment group (target $\mathrm{SBP}<180 \mathrm{mmHg}$; $n=1430$ ). Patients were within $6 \mathrm{~h}$ of onset and average time between symptom onset and treatment initiation was 4 (2.9-5.1) $\mathrm{h}$ and 4.5 (3-7) $\mathrm{h}$, in the intensive and conservative treatment groups, respectively ${ }^{[49]}$. In the intensive treatment group, target SBP was not achieved in the $66.6 \%$ of patients within 1 h. Intensive BP reduction was safe but did not cause a statistical significance in the major disability or death rate, but a secondary analysis of ordinal modified Rankin Scale (mRS) showed that intensive BP reduction led to better physical functional outcomes compared with conservative treatment.

In the CT substudy, which included 964 (40\%) patients of INTERACT-2, baseline and $24 \pm 3$-h CT images of the brain were analyzed ${ }^{[60]}$. Results showed that intensive SBP reduction (target $\mathrm{SBP}<140 \mathrm{mmHg}$ ) was achieved within $1 \mathrm{~h}$ and consistently maintained, and protected against hematoma enlargement for $24 \mathrm{~h}$. Of 491 patients randomized to intensive BP reduction, the least hematoma expansion occurring in patients reached target SBP early ( $\leq 1 \mathrm{~h}, 2.6 \mathrm{~mL} ; 1$ to $6 \mathrm{~h}, 4.7 \mathrm{~mL}$ and $>6 \mathrm{~h}, 5.4 \mathrm{~mL})$. Greater reduction in SBP resulted in less hematoma expansion (for $<10 \mathrm{mmHg}, 13.3 \mathrm{~mL} ; 10-20 \mathrm{mmHg}, 5.0 \mathrm{~mL}$ and $\geq 20 \mathrm{mmHg}$, $3.0 \mathrm{~mL})$. 
In the follow-up analysis of Wang et al. ${ }^{[61]}$, effects of SBP reduction in terms of death or major disability at 90 days over three time periods (15-60 min, 1-24 h, and 2-7 days) after randomization were analyzed. Optimal recovery was associated with the greatest reduction in SBP $(\geq 20 \mathrm{mmHg})$ achieved in the first hour and sustained for 7 days.

In another follow-up analysis, intensive BP reduction found to be beneficial on physical function at 90 days across a wide range of initial SBP levels $(<160,160-169,170-179,180-189$, and $\geq 190 \mathrm{mmHg})$ and SBP level of 130-139 mmHg provided maximum benefit ${ }^{\left[{ }^{[62]}\right.}$. Compared with achieved SBP of $130 \mathrm{mmHg}$, adjusted ORs for poor outcome were 1.21 at $140 \mathrm{mmHg}, 1.42$ at $150 \mathrm{mmHg}, 1.57$ at $160 \mathrm{mmHg}, 2.19$ at $170 \mathrm{mmHg}$, and 3.04 at $180 \mathrm{mmHg}$. Death or major disability rate was slightly increased at $120 \mathrm{mmHg}$ compared with $130 \mathrm{mmHg}$.

\section{Meta-analysis of Koch et al. ${ }^{[52]}$, INTERACT, ICH ADAPT, INTERACT-2, 2014}

This meta-analysis including 3315 acute ICH patients, indicated that intensive BP lowering (target $\mathrm{SBP}<$ $150 \mathrm{mmHg}$ or $\mathrm{MAP}<110 \mathrm{mmHg}$ ) was safe ${ }^{[63]}$. Mortality rates were similar between intensive and guideline $\mathrm{BP}$ treatment groups. Intensive BP reduction lowered 3-month death and dependency (mRS grades 3-6) and absolute hematoma growth at $24 \mathrm{~h}$.

\section{ENOS, 2015}

In the Efficacy of Nitric Oxide in Stroke Trial (ENOS), 4011 stroke patients (ischemic or hemorrhagic,) with SBP $140-220 \mathrm{mmHg}$, within $48 \mathrm{~h}$ of symptom onset were randomized to transdermal glyceryl trinitrate (GTN) treatment $(n=1993)$ or no GTN $(n=2002)$ for one week ${ }^{[64]}$. The average time of randomization was $26 \mathrm{~h}$. Transdermal GTN lowered BP and was found to be safe and potentially beneficial if started within $6 \mathrm{~h}$ of stroke onset. However, the distribution of mRS between two groups did not differ. Subgroup analysis of $629 \mathrm{ICH}$ patients with a mean baseline BP of $172.1 / 93.4 \mathrm{mmHg}$ showed no difference in the $\mathrm{mRS}$ and in the rates of SAEs between GTN $(n=310)$ and no GTN $(n=319)$ groups $^{[65]}$. When adjusted for baseline value, GTN treatment caused a smaller hematoma volume (mean difference, $-4.3 \mathrm{~cm}^{3} ; P=0.06$ ) in 181 patients whose brain imaging repeated one week later. Starting GTN treatment within $6 \mathrm{~h}$ was associated with better outcomes (mRS score, early impairment, late dependency, late cognition, mood, life quality, disability, and death)

\section{Gong et al. ${ }^{[6]}, 2015$}

Gong et al. ${ }^{[66]}$ investigated the effect of BP control in ultra-early ICH. One hundred and twenty ICH patients with SBP $>160 \mathrm{mmHg}$, within $4 \mathrm{~h}$ of symptom onset were divided into strengthened antihypertensive (target SBP 130-140 mmHg; $n=60$ ) and standard antihypertensive (target SBP 160-180 mmHg; $n=60$ ) group. IV nitroglycerin was used. Mean time of treatment initiation after symptom onset in the strengthened and standard treatment group was $2.24 \pm 1.23 \mathrm{~h}$ and $2.13 \pm 1.05 \mathrm{~h}$, respectively. In this study, where treatment was started much earlier compared to others, strengthened treatment significantly lowered hematoma volume and hematoma enlargement after $24 \mathrm{~h}$ and National Institutes of Health Stroke Scale (NIHSS) score on day 14. Additionally, cerebral edema amount and serum matrix metalloproteinase-9 level (related to the prognosis of brain hemorrhage) after 5 days and 14 days were significantly lower in the experimental group ${ }^{[67]}$.

\section{ATACH-2, 2016}

The ATACH-2 trial investigated the effectiveness of rapid SBP lowering in ICH patients with SBP > $180 \mathrm{mmHg}$ (determined on the basis that a greater therapeutic benefit would be shown by excluding patients who did not require IV antihypertensive treatment) in an earlier time window (within $4.5 \mathrm{~h}$ from symptom onset). Patients randomized to intensive treatment (target SBP 110-139 mmHg; $n=500$ ) or 
standard treatment (target SBP 140-179 mmHg; $n=500)^{[48,68]}$. IV nicardipine (first line), labetalol, diltiazem or uradipil were used. Time to start treatment in the intensive and standard treatment groups was $182.2( \pm$ $57.2) \mathrm{min}$ and $184.7( \pm 56.7) \mathrm{min}$, respectively. $12.2 \%$ of patients in the intensive and $0.8 \%$ of patients in the standard treatment group did not reach the target SBP within $2 \mathrm{~h}$ of randomization. The proportion of SAEs within $72 \mathrm{~h}$ was low while the proportion of patients with any SAEs in the first 3 months and renal adverse events within the first week was higher in the intensive treatment group ${ }^{[48]}$.

Recently, post hoc analysis of the ATACH-2 trial results, which analyzed the effect of intensive (target SBP 110-139 mmHg) over standard (target SBP 140-179 mmHg) treatment in 682 moderate to severe grade patients (Glaskow Coma Scale $<13$ or baseline NIHSS $\geq 10$ or presence of intraventricular hemorrhage) has been published ${ }^{\left[{ }^{[\sigma]}\right]}$. According to study results, intensive treatment reduced the hematoma expansion frequency (20.4\% vs. $27.9 \%$ ), but did not reduce the rate of death or disability.

\section{Meta-analysis of Koch et al. ${ }^{[52]}$, INTERACT, INTERACT-2, ADAPT, Gong et al. ${ }^{[66]}$, ATACH-2, 2017}

The meta-analysis reported similar incidence of 3-month SAEs between intensive (target $\mathrm{SBP}<140 \mathrm{mmHg}$ or $\mathrm{MAP}<110 \mathrm{mmHg}$ ) and conservative treatment groups ${ }^{[70]}$. Despite including the ATACH-2 trial in which higher renal adverse events were observed with intensive treatment in the first week, the incidence of $72 \mathrm{~h}$ hypotension was similar in the two groups. Intensive BP reduction did not improve $24 \mathrm{~h}$ neurological recovery or 3-month functional outcomes. Subgroup analysis showed that intensive BP lowering had significant reducing effect on hematoma growth in age $\leq 62$ years, treatment receiving time $\leq 6 \mathrm{~h}$, initial hematoma volume $\leq 15 \mathrm{~mL}$, and combined intraventricular hemorrhage $\leq 25 \%$ subgroups.

\section{Meta-analysis of Koch et al. ${ }^{[52]}$, INTERACT, INTERACT-2, ICH-ADAPT, ATACH-2, 2017}

This study included 2162 and 2188 participants in the intensive and conservative treatment groups, respectively ${ }^{[7]]}$. The mean duration of treatment onset was greater than $3 \mathrm{~h}$ in all trials included in the metaanalysis and target BP could not be achieved within $1 \mathrm{~h}$ in two-thirds of the patients in the intensive treatment group. Early intensive BP reduction was safe and attenuated hematoma expansion, but did not cause differences in 3-month mortality or major disability. Rates of early neurological deterioration, hypotension, severe hypotension within $72 \mathrm{~h}$, recurrent stroke, acute coronary events, or treatmentemergent adverse events within 3 months were similar between two groups, but renal failure proportion was higher in the intensive treatment group (weight: $81.74 \%$ ATACH-2, 18.26\% INTERACT).

\section{RIGHT-2, 2019}

The Rapid Intervention with Glyceryl Trinitrate in Hypertensive Stroke Trial (RIGHT) was an ambulancebased pilot study ${ }^{[72]}$. Transdermal GTN $(5 \mathrm{mg} / 24 \mathrm{~h})$ started by paramedics in probable ultra-acute $(<4 \mathrm{~h})$ stroke patients with SBP $>140 \mathrm{mmHg}$ and continued for 7 days. Only $6 \mathrm{ICH}$ patients enrolled. Results showed that paramedics were able to successfully enroll patients, and GTN was safe and reduced SBP at $2 \mathrm{~h}$ $(153 \pm 31 \mathrm{mmHg} v s .174 \pm 27 \mathrm{mmHg}$ ).

The subsequent RIGHT-2 trial included 1149 patients with SBP $\geq 120 \mathrm{mmHg}$, within $4 \mathrm{~h}$ of symptom onset ${ }^{[73]}$. One hundred and forty-five patients were diagnosed with ICH, of whom 51.03\% received GTN, and $48.97 \%$ received sham. First treatment was administered by the paramedics, and further treatments were given in the hospital for 3 days. Median randomization time was 74 min (interquartile range, 45-110) and mean initial SBP was $176 \pm 27 \mathrm{mmHg}$. BP at admission was lower in the GTN group (mean, $4.4 / 3.5 \mathrm{mmHg}$ ). SAE rate was similar, and the $\mathrm{mRS}$ score at day 90 was nonsignificantly higher in the GTN group. Clinical outcomes including disability, cognition, dependency, life quality, and mood were worse with GTN and death in hospital was increased with GTN. Hematoma size and growth, and midline shift and mass effect on brain imaging were greater in the GTN group. Additionally, GTN was associated with 
altered use of hospital resources.

\section{ICH ADAPT-2, Ongoing}

This study, which is the phase II trial of ICH ADAPT, will assess ischemic lesion development in ICH patients within $6 \mathrm{~h}$ of symptom onset, with a SBP target of $<140 \mathrm{mmHg}$ and $<180 \mathrm{mmHg}^{[74]}$. Patients will be treated with IV labetalol, hydralazine or enalapril and diffusion weighted imaging will be used. The study is not yet complete.

\section{Incorporation of results of randomized clinical trials and meta-analyses into clinical practice issues} Treatment threshold and target blood pressure

The INTERACT trial results indicated intensive BP lowering attenuated hematoma growth, and even target SBP of $<140 \mathrm{mmHg}$ was achieved only in the $42 \%$ and $66 \%$ of patients within $1 \mathrm{~h}$ and $6 \mathrm{~h}$ after randomization, respectively ${ }^{[38]}$. The INTERACT-2 trial included patients with SBP $\geq 150 \mathrm{mmHg}$ and only $33.4 \%$ of patients in the intensive treatment group achieved the target SBP of $<140 \mathrm{mmHg}$ at $1 \mathrm{~h}$ postrandomization and the SBP profile reflected values close to $140 \mathrm{mmHg}^{[49]}$. According to post hoc analysis of INTERACT-2 trial, having an average SBP of $130 \mathrm{mmHg}$ at selected time points within $24 \mathrm{~h}$ after randomization was associated with the lowest probability of death and disability at three months ${ }^{[49]}$. On the other hand, follow-up analysis of INTERACT-2 trial reported increase in the risk of death and major disability with SBP of $120 \mathrm{mmHg}$ compared to $130 \mathrm{mmHg}^{[62]}$. In the ATACH-2 trial, the mean minimum SBP during the first $2 \mathrm{~h}$ was $141.1 \pm 14.8 \mathrm{mmHg}$ and $128.9 \pm 16 \mathrm{mmHg}$ in the standard treatment group and intensive treatment group, respectively. Intensive treatment showed no clinical benefit and, moreover, it was associated with a higher rate of renal adverse events and SAEs within the first 3 months ${ }^{[68]}$.

In the meta-analysis of Koch et al. ${ }^{[52]}$, INTERACT, ICH ADAPT, INTERACT-2 trials, intensive BP-lowering treatment (target $\mathrm{MAP}<110 \mathrm{mmHg}$, or $\mathrm{SBP}<150 \mathrm{mmHg}$ ) reduced 3-month mortality and dependency and provided a greater reduction in hematoma growth at $24 \mathrm{~h}$ compared with guideline treatment ${ }^{[63]}$. In the meta-analysis of Koch et al. ${ }^{[52]}$, INTERACT, INTERACT-2, ADAPT, Gong et al. ${ }^{[6]}$, ATACH-2 trials, intensive BP lowering (target $\mathrm{SBP}<140 \mathrm{mmHg}$ or MAP $<110 \mathrm{mmHg}$ ) showed no effects on $24 \mathrm{~h}$ neurologic improvement and 3-month functional outcome, and only attenuated hematoma growth in patients age $\leq 62$ years with hematoma volume $\leq 15 \mathrm{~mL}$ received treatment within $6 \mathrm{~h}$ after symptom onset $^{[70]}$. In the meta-analysis including Koch et al. ${ }^{[55]}$, INTERACT, INTERACT-2, ICH-ADAPT, ATACH-2 trials, no obvious benefit of intensive BP lowering was observed and the rate of renal failure was higher with intensive treatment [driven predominantly by the trial with lowest SBP target (110-139 $\mathrm{mmHg})]^{[71]}$.

In summary, the results of two phase III trials (ATACH-2 and INTERACT-2) with larger sample sizes than other studies, and a meta-analysis that included these two trials indicated that reaching a target below $130 \mathrm{mmHg}$ may not be beneficial, but may be harmful. Therefore, available evidence supports the safety of a treatment that aims to keep SBP between 130 and $150 \mathrm{mmHg}$. However, due to the risk of precipitating cerebral hypoperfusion, studies are needed to investigate the safety of this target in patients with large hematomas, ICP elevation, and impaired cerebral perfusion pressure.

\section{Therapeutic window}

Although ICH is likely to have a wider therapeutic window due to the absence of ischemic penumbra, therapeutic window in ICH patients is still short, as the hematoma expansion mostly occurs within the first few hours. Recombinant Factor VIIa in Acute Intracerebral Haemorrhage (FAST) trial showed that treatment with rfVIIa reduced the rate of hematoma expansion when initiated within the first $4 \mathrm{~h}$ and reduced even greater if this time was limited to $2.5 \mathrm{~h}^{[20]}$. Data from the ATACH trial also showed that even reduction of SBP within $4.5 \mathrm{~h}$ after symptom onset resulted in lower hematoma expansion and lower 
disability and death rate ${ }^{[54]}$. As the ATACH trial pointed out that hematoma expansion incidence was similar in patients presenting within first 0-3 h and $3-4.5 \mathrm{~h}$, the recruitment time window of ATACH-2 trial which was accepted as $3 \mathrm{~h}$ was extended to $4.5 \mathrm{~h}^{[75]}$. In the ADAPT trial, the effects of intensive BP reduction were similar in patients treated within $3 \mathrm{~h}$ or $4.5 \mathrm{~h}$ after symptom onset ${ }^{[59]}$. Better results were obtained if GTN therapy was started within $6 \mathrm{~h}$ after symptom onset in the ENOS trial ${ }^{[65]}$. Meta-analysis of Koch et al..$^{[52]}$, INTERACT, INTERACT 2, ADAPT, Gong et al. ${ }^{[6]]}$, ATACH-2 trials also showed that intensive BP lowering reduced hematoma growth if the treatment was started within the first $6 \mathrm{~h}$ after symptom onset ${ }^{[70]}$. Therefore, a treatment window of the first $6 \mathrm{~h}$ is supported by current evidence to provide therapeutic benefit. However, it is not yet clear when we should start the treatment at the earliest. In the RIGHT-2 trial, in which the median randomization time was shortest compared to other clinical studies (see Table 1), ultra-acute use of transdermal GTN (within $2 \mathrm{~h}$ after symptom onset) worsened the outcome. On the other hand, the same treatment improved functional outcome if started within the first $6 \mathrm{~h}$, in the ENOS trial ${ }^{[65]}$. Differences in treatment duration, sample size, premorbid dependence, and hematoma size in the two studies may be responsible for the different results. However, it is also possible that starting treatment too early may be responsible. After all, the first step of hemostasis, vasoconstriction is prevented with GTN treatment. Further randomized evidence from studies comparing the use of GTN and other agents in the ultra-acute period is needed to understand whether GTN or timing was responsible.

\section{Antihypertensive agents}

Variable antihypertensive agents (with different mechanisms and side-effect profiles) were used in the clinical trials, even within the same study (see Tables 1 and 2) which may have contributed to the final outcome differences. Currently, there is no consensus about the best drugs for BP reduction in the acute setting of ICH and current guidelines recommends individualized approach. The choice should be based on few principles, including ease of titration, rapidity of onset, lack of fluctuations on CBF, lack of negative effect on platelet activity and better controllability. IV drugs should be agents of choice, as oral agents take hours to days to achieve adequate effects, and sublingual agents may cause fast, unpredictable decreases in systemic BP. Direct acting arteriolar or venous vasodilators such as nitroglycerine, sodium nitroprusside, hydralazine shouldn't be the first-line agents and should be used with caution, as vasodilation can increase cerebral blood volume and lead to increased ICP. Appropriate fluid repletion is required prior to pharmacological intervention to avoid greater than expected reduction in SBP during initiation of IV antihypertensive agents. Adequate SBP control should be maintained by infusion for $24 \mathrm{~h}$ (24-27 h after symptom onset) as hematoma expansion often occurs during this period ${ }^{[76]}$.

Transdermal administration was thought to be a safe option, due to presence of severe dysphagia in most stroke patients and effects of transdermal GTN investigated in the clinical trials. However, the ENOS trial which found transdermal GTN was associated with better outcomes if started $<6 \mathrm{~h}$, comparing patients who received GTN with those who did not. There is no evidence that it is superior to IV agents. Furthermore, GTN worsened outcomes in the RIGHT-2 trial, where it started within $2 \mathrm{~h}$ after symptom onset and results showed that the use of GTN should be avoided in the prehospital setting. The possibility of other nitrovasodilators causing similar results should not be excluded.

Another clinical problem is whether the antihypertensive drugs that patients take regularly should be continued or stopped during the acute phase after ICH. Approximately 50\% of stroke patients present while on regular antihypertensive therapy ${ }^{[35]}$. Because of dysphagia and unpredictable responses to IV antihypertensive medications, it is reasonable to temporarily discontinue or reduce these medications at the onset of acute ICH. On the other hand, continuing antihypertensive drugs may contribute to preventing the occurrence of antihypertensive withdrawal syndrome, especially seen with $\beta$-blocker discontinuation. In the 
Table 2. Pharmacological characteristics of intravenous and transdermal antihypertensive agents used in acute blood pressure management in intracerebral hemorrhage patients in clinical trials

\begin{tabular}{|c|c|c|c|c|c|c|c|c|c|}
\hline Agent & Mechanism of action & Intravenous dose & Onset & Half-life & CBF & ICP & Autoregulation & $\begin{array}{l}\text { Platelet } \\
\text { activity }\end{array}$ & Trial name \\
\hline Labetalol $^{[13,17,77,78]}$ & $\alpha_{1}, \beta_{1}$, and $\beta_{2}$-receptor blocker & 5-20 mg bolus every $15 \mathrm{~min}$ & $1-2 \min$ & $9 \min$ & $\ldots$ & $\ldots$ & $\ldots$ & - & $\begin{array}{l}\text { INTERACT }^{[38]}, \text { INTERACT-2 }{ }^{[49]}, \\
\text { ATACH- }{ }^{[48]}, \text { Koch et al. }{ }^{[52]}\end{array}$ \\
\hline Metoprolol $^{[77,78]}$ & Selective $\beta_{1}$-receptor blocker & $20-80 \mathrm{mg}$ every $10 \mathrm{~min}$ & 5-10 min & $3-7 h$ & $\ldots$ & $\ldots$ & $\ldots$ & - & INTERACT, INTERACT- $2^{[38,49]}$ \\
\hline Esmolol $^{[17]}$ & Selective $\beta_{1}$-receptor blocker & $\begin{array}{l}250 \mu \mathrm{g} / \mathrm{kg} \text { bolus followed by } 25 \\
\text { to } 300 \mu \mathrm{g} / \mathrm{kg} / \mathrm{min}\end{array}$ & $1-2 \mathrm{~min}$ & $9 \min$ & $\ldots$ & $\ldots$ & $\ldots$ & + & INTERACT- $2^{[49]}$ \\
\hline Urapidil $^{[79,80]}$ & $\begin{array}{l}\text { Peripheral } \alpha 1 \text {-receptor antagonist } \\
\text { and central serotonin } 1 \mathrm{~A} \text { receptor } \\
\text { stimulator }\end{array}$ & $\begin{array}{l}12.5-25 \mathrm{mg} \text { bolus followed by } 5- \\
40 \mathrm{mg} / \mathrm{h} \text { infusion }\end{array}$ & $3-5 \min$ & $2-4.8 \mathrm{~h}$ & $\ldots$ & $\ldots$ & & - & $\begin{array}{l}\text { INTERACT, INTERACT-2, } \\
\text { ATACH- } 2^{[38,48,49]}\end{array}$ \\
\hline Phentolamine ${ }^{[81,82]}$ & $\begin{array}{l}\text { Peripheral nonselective } \alpha \text {-receptor } \\
\text { antagonist }\end{array}$ & $1-5 \mathrm{mg}$ bolus every $5-10 \mathrm{~min}$ & $1-2 \min$ & $19 \mathrm{~min}$ & & $\ldots$ & $\ldots$ & - & INTERACT, INTERACT-2 $2^{[38,49]}$ \\
\hline Clonidine $^{[83]}$ & Central $\alpha_{2}$-receptor agonist & $1.2-7.2 \mu \mathrm{g} / \mathrm{min}$ & 5-10 min & $12 \mathrm{~h}$ & $\ldots$ & $\ldots$ & $\ldots$ & $\ldots$ & INTERACT- $2^{[49]}$ \\
\hline Sodium nitroprusside ${ }^{[17,93]}$ & $\begin{array}{l}\text { Mixed arterial/venous vasodilator } \\
\text { (nitric oxide donor) }\end{array}$ & $0.25-4 \mu \mathrm{g} / \mathrm{kg} / \mathrm{min}$ & $1-2 \mathrm{~min}$ & $<10 \min$ & ++ & ++ & - & - & $\begin{array}{l}\text { INTERACT } \\
\text { Koch et al. }{ }^{[52]} \text {, INTERACT-2 }{ }^{[49],}\end{array}$ \\
\hline Nitroglycerine $^{[17]}$ & $\begin{array}{l}\text { Mixed arterial/venous vasodilator } \\
\text { (nitric oxide donor) }\end{array}$ & $20-400 \mu \mathrm{g} / \mathrm{min}$ & $1-2 \mathrm{~min}$ & $3-5 \min$ & + & $\ldots$ & $\ldots$ & - & $\begin{array}{l}\text { INTERACT }{ }^{[38]} \text {, INTERACT-2 }{ }^{[49]} \text {, } \\
\text { Gong et al. }{ }^{[66]}{ }^{3}\end{array}$ \\
\hline $\begin{array}{l}\text { Transdermal glyceryl } \\
\text { trinitrate }^{[64,84-87]}\end{array}$ & $\begin{array}{l}\text { Mixed arterial/venous vasodilator } \\
\text { (nitric oxide donor) }\end{array}$ & $0.2 \mathrm{mg} / \mathrm{h}, 5 \mathrm{mg} / 24 \mathrm{~h}$ & $15-30 \mathrm{~min}$ & Up to $24 \mathrm{~h}$ & $\ldots$ & $\ldots$ & $\ldots$ & $\ldots$ & $\begin{array}{l}\text { INTERACT, ENOS, RIGHT, } \\
\text { RIGHT- } 2{ }_{\left[33^{\prime} 8,64,72,73^{\prime}\right]}\end{array}$ \\
\hline Nicardipine ${ }^{[13,88]}$ & Calcium channel blocker & $5-15 \mathrm{mg} / \mathrm{h}$ & $5-10 \mathrm{~min}$ & $2-4 \mathrm{~h}$ & + & $\ldots$ & - & - & 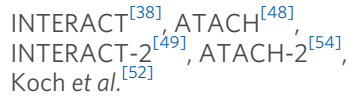 \\
\hline Diltiazem $^{[88,89]}$ & Calcium channel blocker & $\begin{array}{l}0.25 \mathrm{mg} / \mathrm{kg} \text { bolus followed by } 5- \\
15 \mathrm{mg} / \mathrm{h} \text { infusion }\end{array}$ & $2-7 \min$ & $3.4 \mathrm{~h}$ & & $\ldots$ & & - & ATACH-2 $2^{[54]}$ \\
\hline Hydralazine $^{[17]}$ & Arterial vasodilator & 5-20 mg bolus every $15 \mathrm{~min}$ & $5-20 \mathrm{~min}$ & $2-8 \mathrm{~h}$ & ++ & ++ & - & - & $\begin{array}{l}\text { INTERACT, ICH ADAPT, } \\
\text { INTERACT-2 } 2^{[3,49,59]}\end{array}$ \\
\hline Enalapril $^{[17]}$ & ACE inhibitor & $1.25-5 \mathrm{mg}$ every $6 \mathrm{~h}$ & $15-30 \mathrm{~min}$ & $11 \mathrm{~h}$ & & $\ldots$ & ++ & - & \\
\hline $\begin{array}{l}\text { Diuretics (furosemide, } \\
\text { bumetanide) }\end{array}$ & $\begin{array}{l}\text { Inhibits reabsorption of } \mathrm{Na} / \mathrm{Cl} \text { in the } \\
\text { ascending loop of Henle }\end{array}$ & $\begin{array}{l}\text { Furosemide: } 20-40 \mathrm{mg} \text { bolus } \\
\text { followed by } 10-40 \mathrm{mg} / \mathrm{h} \\
\text { Bumetanide: } 0.5-1 \mathrm{mg} / \text { dose up to } \\
2 \text { times a day followed by } \\
0.5-2 \mathrm{mg} / \mathrm{h}\end{array}$ & $\begin{array}{l}\text { Furosemide: } \\
5 \text { min } \\
\text { Bumetanide: } \\
2-3 \text { min }\end{array}$ & $\begin{array}{l}\text { Furosemide: } \\
30-60 \text { min } \\
\text { Bumetanide: 1- } \\
1.5 \mathrm{~h}\end{array}$ & $\ldots / \ldots$ & $\ldots /$. & $\ldots / \ldots$ & $-/-$ & INTERACT, INTERACT- $2^{[38,49]}$ \\
\hline
\end{tabular}

ACE: Angiotensin-converting enzyme; CBF: cerebral blood flow; ICP: intracranial pressure; +: increase or favorable effects; ++: subctantial increase or favorable effects; -: decrease or negative effects; ...: no documented direct effect. 
ENOS trial, a subset of patients who were taking antihypertensive drugs before their stroke were randomly assigned to either continue or stop taking these drugs, and no evidence was found to support continuing pre-stroke antihypertensive drugs in the first few days ${ }^{[64]}$. To date, there is no evidence to support continuing or discontinuing pre-stroke antihypertensive drugs in patients within the first few days after ICH. However, even if they are discontinued, oral antihypertensive agents should be started as soon as possible (preferably 24 to $48 \mathrm{~h}$ after symptom onset, because most of the acute processes are uncommon after the first $24 \mathrm{~h}$ ) to control resistant hypertension and facilitate the transition to long-term management for secondary prophylaxis.

\section{CONCLUSION}

While current trials have shown that intensive BP lowering is clinically feasible and potentially safe, the treatment threshold, BP target, therapeutic window, medications to be used and whether such treatment improves clinical outcomes remain unclear. Current guidelines cannot provide clear answers to these uncertainties. Almost all randomized clinical trials included patients receiving anticoagulant or antiplatelets with different rates and different criteria (while a platelet count $<100.000 \mathrm{~mm}^{3}$ was required to be included in the ATACH study, $<50.000 \mathrm{~mm}^{3}$ was required in the ATACH-2 study; while international normalize ratio $<1.8$ was required to include patients receiving warfarin in the study of Koch et al. $.^{[52]},<1.4$ was required in the ATACH study, etc). The small sample sizes and heterogeneous criteria make it difficult to understand whether we should make individual changes in the treatment of these patients' acute hypertensive response $\mathrm{A}^{[4,52,58]}$. The safety of the current SBP reduction therapies in patients with severe disease, large hematomas, ICP elevation, and impaired cerebral perfusion pressure is unknown as they were not represented in clinical trials. The therapeutic target for SBP lowering may vary between individual patients, depending on individual cerebral hemodynamics. An example is the investigation of the safety and effectiveness of intensive BP lowering in ICH patients with cerebrovascular stenosis ${ }^{[50]}$. The results of future studies may reveal the need for an individualized treatment with CBF and regional ICP measurements.

\section{DECLARATIONS}

\section{Authors' contributions}

Wrote the first draft of the manuscript: Akinci Y

Discussed the results and commented on the manuscript at all stages: Qureshi AI

Read and approved the final manuscript: Akinci Y, Qureshi AI

\section{Availability of data and materials}

Not applicable.

\section{Financial support and sponsorship}

None.

\section{Conflicts of interest}

Both authors declared that there are no conflicts of interest.

\section{Ethical approval and consent to participate}

Not applicable.

\section{Consent for publication}

Not applicable. 


\section{Copyright}

(c) The Author(s) 2021.

\section{REFERENCES}

1. Rajashekar D, Liang JW. Intracerebral hemorrhage. In: StatPearls [Internet]. Treasure Island (FL): StatPearls Publishing; 2021. PubMed

2. Qureshi AI, Mendelow AD, Hanley DF. Intracerebral haemorrhage. Lancet 2009;373:1632-44. DOI PubMed PMC

3. Ziai WC, Carhuapoma JR. Intracerebral hemorrhage. Continuum (Minneap Minn) 2018;24:1603-22. DOI PubMed

4. McEvoy AW, Kitchen ND, Thomas DG. Lesson of the week: intracerebral haemorrhage in young adults: the emerging importance of drug misuse. BMJ 2000;320:1322-4. DOI PubMed PMC

5. Aguilar MI, Freeman WD. Spontaneous intracerebral hemorrhage. Semin Neurol 2010;30:555-64. DOI PubMed

6. Qureshi AI, Qureshi MH. Acute hypertensive response in patients with intracerebral hemorrhage pathophysiology and treatment. $J$ Cereb Blood Flow Metab 2018;38:1551-63. DOI PubMed PMC

7. Davis SM, Broderick J, Hennerici M, et al; Recombinant Activated Factor VII Intracerebral Hemorrhage Trial Investigators. Hematoma growth is a determinant of mortality and poor outcome after intracerebral hemorrhage. Neurology 2006;66:1175-81. DOI PubMed

8. Majidi S, Suarez JI, Qureshi AI. Management of acute hypertensive response in intracerebral hemorrhage patients after ATACH-2 trial. Neurocrit Care 2017;27:249-58. DOI PubMed

9. Terayama Y, Tanahashi N, Fukuuchi Y, Gotoh F. Prognostic value of admission blood pressure in patients with intracerebral hemorrhage. Keio Cooperative Stroke Study. Stroke 1997;28:1185-8. DOI PubMed

10. Fujii Y, Takeuchi S, Sasaki O, Minakawa T, Tanaka R. Multivariate analysis of predictors of hematoma enlargement in spontaneous intracerebral hemorrhage. Stroke 1998;29:1160-6. DOI PubMed

11. Ohwaki K, Yano E, Nagashima H, Hirata M, Nakagomi T, Tamura A. Blood pressure management in acute intracerebral hemorrhage: relationship between elevated blood pressure and hematoma enlargement. Stroke 2004;35:1364-7. DOI PubMed

12. Sang YH, Su HX, Wu WT, So KF, Cheung RT. Elevated blood pressure aggravates intracerebral hemorrhage-induced brain injury. $J$ Neurotrauma 2011;28:2523-34. DOI PubMed PMC

13. Dastur CK, Yu W. Current management of spontaneous intracerebral haemorrhage. Stroke Vasc Neurol 2017;2:21-9. DOI PubMed PMC

14. Biffi A, Anderson CD, Battey TW, et al. Association between blood pressure control and risk of recurrent intracerebral hemorrhage. JAMA 2015;314:904-12. DOI PubMed PMC

15. Qureshi AI, Ezzeddine MA, Nasar A, et al. Prevalence of elevated blood pressure in 563,704 adult patients with stroke presenting to the ED in the United States. Am J Emerg Med 2007;25:32-8. DOI PubMed PMC

16. Brott T, Thalinger K, Hertzberg V. Hypertension as a risk factor for spontaneous intracerebral hemorrhage. Stroke 1986;17:1078-83. DOI PubMed

17. Qureshi AI. Acute hypertensive response in patients with stroke: pathophysiology and management. Circulation 2008;118:176-87. DOI PubMed

18. Fischer U, Cooney MT, Bull LM, et al. Acute post-stroke blood pressure relative to premorbid levels in intracerebral haemorrhage versus major ischaemic stroke: a population-based study. Lancet Neurol 2014;13:374-84. DOI PubMed PMC

19. Kazui S, Minematsu K, Yamamoto H, Sawada T, Yamaguchi T. Predisposing factors to enlargement of spontaneous intracerebral hematoma. Stroke 1997;28:2370-5. DOI PubMed

20. Mayer SA, Brun NC, Begtrup K, et al; FAST Trial Investigators. Efficacy and safety of recombinant activated factor VII for acute intracerebral hemorrhage. N Engl J Med 2008;358:2127-37. DOI PubMed

21. de Gasparo M, Speth RC, Baltatu OC, Vanderheyden P. Brain RAS: hypertension and beyond. Int J Hypertens 2013;2013:157180. DOI PubMed PMC

22. Morishita R, Higaki J, Okunishi H, et al. Role of tissue renin angiotensin system in two-kidney, one-clip hypertensive rats. Am J Physiol 1993;264:F510-4. DOI PubMed

23. Kamińska M, Mogielnicki A, Stankiewicz A, Kramkowski K, Domaniewski T, Buczko W, Chabielska E. Angiotensin II via AT1 receptor accelerates arterial thrombosis in renovascular hypertensive rats. J Physiol Pharmacol 2005;56:571-85. PubMed

24. Wakisaka Y, Chu Y, Miller JD, Rosenberg GA, Heistad DD. Critical role for copper/zinc-superoxide dismutase in preventing spontaneous intracerebral hemorrhage during acute and chronic hypertension in mice. Stroke 2010;41:790-7. DOI PubMed PMC

25. Wakisaka Y, Chu Y, Miller JD, Rosenberg GA, Heistad DD. Spontaneous intracerebral hemorrhage during acute and chronic hypertension in mice. J Cereb Blood Flow Metab 2010;30:56-69. DOI PubMed PMC

26. Ito H, Takemori K, Suzuki T. Role of angiotensin II type 1 receptor in the leucocytes and endothelial cells of brain microvessels in the pathogenesis of hypertensive cerebral injury. J Hypertens 2001;19:591-7. DOI PubMed

27. Lanz TV, Ding Z, Ho PP, et al. Angiotensin II sustains brain inflammation in mice via TGF-beta. J Clin Invest 2010;120:2782-94. DOI PubMed PMC

28. Wosik K, Cayrol R, Dodelet-Devillers A, et al. Angiotensin II controls occludin function and is required for blood brain barrier maintenance: relevance to multiple sclerosis. J Neurosci 2007;27:9032-42. DOI PubMed PMC

29. Miyoshi M, Miyano K, Moriyama N, Taniguchi M, Watanabe T. Angiotensin type 1 receptor antagonist inhibits lipopolysaccharideinduced stimulation of rat microglial cells by suppressing nuclear factor kappaB and activator protein-1 activation. Eur J Neurosci 
2008;27:343-51. DOI PubMed

30. Ando H, Zhou J, Macova M, Imboden H, Saavedra JM. Angiotensin II AT1 receptor blockade reverses pathological hypertrophy and inflammation in brain microvessels of spontaneously hypertensive rats. Stroke 2004;35:1726-31. DOI PubMed

31. Tanaka J, Hayashi Y, Watai T, Hori K, Nomura M. Noradrenaline release in the rat subfornical organ area to blood pressure changes. Exp Neurol 1998;152:303-6. DOI PubMed

32. Iwata M, Inoue S, Kawaguchi M, Nakamura M, Konishi N, Furuya H. Posttreatment but not pretreatment with selective betaadrenoreceptor 1 antagonists provides neuroprotection in the hippocampus in rats subjected to transient forebrain ischemia. Anesth Analg 2010;110:1126-32. DOI PubMed

33. Goyagi T, Kimura T, Nishikawa T, Tobe Y, Masaki Y. Beta-adrenoreceptor antagonists attenuate brain injury after transient focal ischemia in rats. Anesth Analg 2006;103:658-63. DOI PubMed

34. Benveniste H, Kim KR, Hedlund LW, Kim JW, Friedman AH. Cerebral hemorrhage and edema following brain biopsy in rats: significance of mean arterial blood pressure. J Neurosurg 2000;92:100-7. DOI PubMed

35. Bath P, Chalmers J, Powers W, et al; International Society of Hypertension Writing Group. International Society of Hypertension (ISH): statement on the management of blood pressure in acute stroke. J Hypertens 2003;21:665-72. DOI PubMed

36. Quiñones-ossa GA, Durango-espinosa Y, Padilla-zambrano H, et al. The puzzle of spontaneous versus traumatic intracranial hemorrhages. Egypt J Neurosurg 2020:35. DOI

37. Dandapani BK, Suzuki S, Kelley RE, Reyes-Iglesias Y, Duncan RC. Relation between blood pressure and outcome in intracerebral hemorrhage. Stroke 1995;26:21-4. DOI PubMed

38. Anderson CS, Huang Y, Wang JG, et al. Intensive blood pressure reduction in acute cerebral haemorrhage trial (INTERACT): a randomised pilot trial. Lancet Neurol 2008;7:391-9. DOI PubMed

39. Hemphill JC 3rd, Greenberg SM, Anderson CS, et al; American Heart Association Stroke Council; Council on Cardiovascular and Stroke Nursing; Council on Clinical Cardiology. Guidelines for the management of spontaneous intracerebral hemorrhage: a guideline for healthcare professionals from the american heart association/american stroke association. Stroke 2015;46:2032-60. DOI PubMed

40. Steiner T, Al-Shahi Salman R, Beer R, et al; European Stroke Organisation. European Stroke Organisation (ESO) guidelines for the management of spontaneous intracerebral hemorrhage. Int J Stroke 2014;9:840-55. DOI PubMed

41. Strandgaard S. Autoregulation of cerebral blood flow in hypertensive patients. The modifying influence of prolonged antihypertensive treatment on the tolerance to acute, drug-induced hypotension. Circulation 1976;53:720-7. DOI PubMed

42. Xie X, Xu J, Gu H, et al. The J-curve association between systolic blood pressure and clinical outcomes in ischemic stroke or TIA: the BOSS study. Sci Rep 2017;7:14023. DOI PubMed PMC

43. Vemmos KN, Tsivgoulis G, Spengos K, et al. U-shaped relationship between mortality and admission blood pressure in patients with acute stroke. J Intern Med 2004;255:257-65. DOI PubMed

44. Qureshi AI, Bliwise DL, Bliwise NG, Akbar MS, Uzen G, Frankel MR. Rate of 24-hour blood pressure decline and mortality after spontaneous intracerebral hemorrhage: a retrospective analysis with a random effects regression model. Crit Care Med 1999;27:480-5. DOI PubMed

45. Zazulia AR, Diringer MN, Videen TO, et al. Hypoperfusion without ischemia surrounding acute intracerebral hemorrhage. $J$ Cereb Blood Flow Metab 2001;21:804-10. DOI PubMed

46. Kim-Han JS, Kopp SJ, Dugan LL, Diringer MN. Perihematomal mitochondrial dysfunction after intracerebral hemorrhage. Stroke 2006;37:2457-62. DOI PubMed

47. Powers WJ, Zazulia AR, Videen TO, et al. Autoregulation of cerebral blood flow surrounding acute (6 to 22 hours) intracerebral hemorrhage. Neurology 2001;57:18-24. DOI PubMed

48. Qureshi AI, Palesch YY, Barsan WG, et al; ATACH-2 Trial Investigators and the Neurological Emergency Treatment Trials Network. Intensive blood-pressure lowering in patients with acute cerebral hemorrhage. N Engl J Med 2016;375:1033-43. DOI PubMed PMC

49. Anderson CS, Heeley E, Huang Y, et al; INTERACT2 Investigators. Rapid blood-pressure lowering in patients with acute intracerebral hemorrhage. N Engl J Med 2013;368:2355-65. DOI PubMed

50. Ye Z, Ai X, Zheng J, et al. Antihypertensive treatments for spontaneous intracerebral hemorrhage in patients with cerebrovascular stenosis: a randomized clinical trial (ATICHST). Medicine (Baltimore) 2017;96:e7289. DOI PubMed PMC

51. Anderson CS, Huang Y, Arima H, et al; INTERACT Investigators. Effects of early intensive blood pressure-lowering treatment on the growth of hematoma and perihematomal edema in acute intracerebral hemorrhage: the Intensive Blood Pressure Reduction in Acute Cerebral Haemorrhage Trial (INTERACT). Stroke 2010;41:307-12. DOI PubMed

52. Koch S, Romano JG, Forteza AM, Otero CM, Rabinstein AA. Rapid blood pressure reduction in acute intracerebral hemorrhage: feasibility and safety. Neurocrit Care 2008;8:316-21. DOI PubMed

53. Arima H, Anderson CS, Wang JG, et al; Intensive Blood Pressure Reduction in Acute Cerebral Haemorrhage Trial Investigators. Lower treatment blood pressure is associated with greatest reduction in hematoma growth after acute intracerebral hemorrhage. Hypertension 2010;56:852-8. DOI PubMed

54. Qureshi AI, Palesch YY, Martin R, et al; Antihypertensive Treatment of Acute Cerebral Hemorrhage Study Investigators. Effect of systolic blood pressure reduction on hematoma expansion, perihematomal edema, and 3-month outcome among patients with intracerebral hemorrhage: results from the antihypertensive treatment of acute cerebral hemorrhage study. Arch Neurol 2010;67:570-6. DOI PubMed PMC

55. Steiner T, Kaste M, Forsting M, et al. Recommendations for the management of intracranial haemorrhage - part I: spontaneous intracerebral haemorrhage. The European stroke initiative writing committee and the writing committee for the EUSI executive committee. Cerebrovasc Dis 2006;22:294-316. DOI PubMed 
56. Spence JD, Del Maestro RF. Hypertension in acute ischemic strokes. Treat. Arch Neurol 1985;42:1000-2. DOI PubMed

57. Qureshi AI. The importance of acute hypertensive response in ICH. Stroke 2013;44:S67-9. DOI PubMed

58. Treatment of Acute Cerebral Hemorrhage (ATACH) investigators. Antihypertensive treatment of acute cerebral hemorrhage. Crit Care Med 2010;38:637-48. DOI PubMed PMC

59. Butcher KS, Jeerakathil T, Hill M, et al; ICH ADAPT Investigators. The intracerebral hemorrhage acutely decreasing arterial pressure trial. Stroke 2013;44:620-6. DOI PubMed

60. Carcel C, Wang X, Sato S, et al; INTERACT2 Investigators. Degree and timing of intensive blood pressure lowering on hematoma growth in intracerebral hemorrhage: intensive blood pressure reduction in acute cerebral hemorrhage trial-2 results. Stroke 2016;47:1651-3. DOI PubMed

61. Wang X, Arima H, Heeley E, et al; INTERACT2 Investigators. Magnitude of blood pressure reduction and clinical outcomes in acute intracerebral hemorrhage: intensive blood pressure reduction in acute cerebral hemorrhage trial study. Hypertension 2015;65:1026-32. DOI PubMed

62. Arima H, Heeley E, Delcourt C, et al; INTERACT2 Investigators, INTERACT2 Investigators. Optimal achieved blood pressure in acute intracerebral hemorrhage: INTERACT2. Neurology 2015;84:464-71. DOI PubMed PMC

63. Tsivgoulis G, Katsanos AH, Butcher KS, et al. Intensive blood pressure reduction in acute intracerebral hemorrhage: a meta-analysis. Neurology 2014;83:1523-9. DOI PubMed

64. ENOS Trial Investigators. Efficacy of nitric oxide, with or without continuing antihypertensive treatment, for management of high blood pressure in acute stroke (ENOS): a partial-factorial randomised controlled trial. Lancet 2015;385:617-28. DOI PubMed PMC

65. Krishnan K, Scutt P, Woodhouse L, et al. Glyceryl trinitrate for acute intracerebral hemorrhage: results from the efficacy of nitric oxide in stroke (ENOS) trial, a subgroup analysis. Stroke 2016;47:44-52. DOI PubMed

66. Gong FT, Yu LP, Gong YH, Zhang YX, Wang ZG, Yan CZ. Blood pressure control in ultra-early basal ganglia intracerebral hemorrhage. Eur Rev Med Pharmacol Sci 2015;19:412-5. PubMed

67. Dang B, Duan X, Wang Z, He W, Chen G. A therapeutic target of cerebral hemorrhagic stroke: matrix metalloproteinase-9. Curr Drug Targets 2017;18:1358-66. DOI PubMed

68. Qureshi AI, Palesch YY. Antihypertensive treatment of acute cerebral hemorrhage (ATACH) II: design, methods, and rationale. Neurocrit Care 2011;15:559-76. DOI PubMed PMC

69. Qureshi AI, Foster LD, Lobanova I, Huang W, Suarez JI. Intensive blood pressure lowering in patients with moderate to severe grade acute cerebral hemorrhage: post hoc analysis of antihypertensive treatment of acute cerebral hemorrhage (ATACH)-2 trial. Cerebrovasc Dis 2020;49:244-52. DOI PubMed

70. Shi L, Xu S, Zheng J, Xu J, Zhang J. Blood pressure management for acute intracerebral hemorrhage: a meta-analysis. Sci Rep 2017;7:14345. DOI PubMed PMC

71. Lattanzi S, Cagnetti C, Provinciali L, Silvestrini M. How should we lower blood pressure after cerebral hemorrhage? Cerebrovasc Dis 2017;43:207-13. DOI PubMed

72. Ankolekar S, Fuller M, Cross I, et al. Feasibility of an ambulance-based stroke trial, and safety of glyceryl trinitrate in ultra-acute stroke: the rapid intervention with glyceryl trinitrate in Hypertensive Stroke Trial (RIGHT, ISRCTN66434824). Stroke 2013;44:31208. DOI PubMed

73. Bath PM, Woodhouse LJ, Krishnan K, et al. Prehospital transdermal glyceryl trinitrate for ultra-acute intracerebral hemorrhage: data from the RIGHT-2 trial. Stroke 2019;50:3064-71. DOI PubMed PMC

74. Gioia L, Klahr A, Kate M, et al. The intracerebral hemorrhage acutely decreasing arterial pressure trial II (ICH ADAPT II) protocol. BMC Neurol 2017;17:100. DOI PubMed PMC

75. Qureshi A, Palesch Y; ATACH II Investigators. Expansion of recruitment time window in antihypertensive treatment of acute cerebral hemorrhage (ATACH) II trial. J Vasc Interv Neurol 2012;5:6-9. PubMed PMC

76. Qureshi AI. Intracerebral hemorrhage specific intensity of care quality metrics. Neurocrit Care 2011;14:291-317. DOI PubMed

77. Griffith DN, James IM, Newbury PA, Woollard ML. The effect of beta-adrenergic receptor blocking drugs on cerebral blood flow. $\mathrm{Br}$ J Clin Pharmacol 1979;7:491-4. DOI PubMed PMC

78. Bonten TN, Plaizier CE, Snoep JJ, Stijnen T, Dekkers OM, van der Bom JG. Effect of $\beta$-blockers on platelet aggregation: a systematic review and meta-analysis. Br J Clin Pharmacol 2014;78:940-9. DOI PubMed PMC

79. Puchstein C, Van Aken H, Anger C, Hidding J. Influence of urapidil on intracranial pressure and intracranial compliance in dogs. $\mathrm{Br} J$ Anaesth 1983;55:443-8. DOI PubMed

80. Storck J, Ochs JG, Kirsten R. Effects of urapidil on 5-hydroxytryptamine induced platelet aggregation and on 14C-5hydroxytryptamine uptake in platelets. Int J Clin Pharmacol Ther Toxicol 1990;28:303-8. PubMed

81. Spalding A, Vaitkevicius H, Dill S, MacKenzie S, Schmaier A, Lockette W. Mechanism of epinephrine-induced platelet aggregation. Hypertension 1998;31:603-7. DOI PubMed

82. Anfossi G, Trovati M, Mularoni E, Lanzio M, Massucco P, Emanuelli G. Influence of phentolamine on platelet aggregation, thromboxane B2 production and release reaction. Arch Int Pharmacodyn Ther 1988;296:144-54. PubMed

83. Akpek EA, Kahraman S, Yaman A, Bulutçu E, Yavuzer S, Ozgen S. The effect of pre-induction clonidine on platelet aggregation during minor surgery. Eur J Clin Pharmacol 1998;54:459-62. DOI PubMed

84. Willmot M, Ghadami A, Whysall B, Clarke W, Wardlaw J, Bath PM. Transdermal glyceryl trinitrate lowers blood pressure and maintains cerebral blood flow in recent stroke. Hypertension 2006;47:1209-15. DOI PubMed

85. Butterworth RJ, Cluckie A, Jackson SH, Buxton-Thomas M, Bath PM. Pathophysiological assessment of nitric oxide (given as sodium nitroprusside) in acute ischaemic stroke. Cerebrovasc Dis 1998;8:158-65. DOI PubMed 
86. Bath PM, Pathansali R, Iddenden R, Bath FJ. The effect of transdermal glyceryl trinitrate, a nitric oxide donor, on blood pressure and platelet function in acute stroke. Cerebrovasc Dis 2001;11:265-72. DOI PubMed

87. Bath PM, Scutt P, Anderson CS, et al. Prehospital transdermal glyceryl trinitrate in patients with ultra-acute presumed stroke (RIGHT2): an ambulance-based, randomised, sham-controlled, blinded, phase 3 trial. Lancet 2019;393:1009-20. DOI PubMed PMC

88. Abe K, Iwanaga H, Inada E. Effect of nicardipine and diltiazem on internal carotid artery blood flow velocity and local cerebral blood flow during cerebral aneurysm surgery for subarachnoid hemorrhage. J Clin Anesth 1994;6:99-105. DOI PubMed

89. Pearce WJ, Bevan JA. Diltiazem and autoregulation of canine cerebral blood flow. J Pharmacol Exp Ther 1987;242:812-7. PubMed

90. Walley TJ, Woods KL, Barnett DB. Effects of calcium channel blockers on in vitro platelet function in whole blood using single platelet counting. Thromb Haemost 1989;61:137-9. PubMed

91. Plangger C, Völkl H. Effect of furosemide, bumetanide and mannitol on intracranial pressure in experimental brain edema of the rat. Zentralbl Neurochir 1989;50:142-4. PubMed

92. Chohan IS, Singh I, Vermylen J. Furosemide: its role on certain platelet functions. Acta Clin Belg 1977;32:39-43. DOI PubMed 